\title{
Non-invasive in vivo methods to measure lipidic formulae efficacy at the skin surface: advantages and limits
}

\author{
Elodie Prestat-Marquis ${ }^{*}$ \\ Eurofins EVIC France, 57 rue Ulysse Gayon, Bordeaux, France
}

Received 18 July 2018 - Accepted 6 September 2018

\begin{abstract}
Lipids are major ingredients in cosmetics and play an essential role in cosmetics ability to improve skin, hair, nail and mucuous membranes aspect, sensory qualities and function, either by interacting with skin components, enhancing active ingredient release or by spreading and forming a protective layer on its surface. Measuring lipids efficacy to the skin is possible through a wide range of non-invasive clinical and instrumental methods. Measurements of sebum, temperature, hydration, transepidermal water loss, skin surface topography, skin firmness, elasticity, color enable to assess the lipids ability to protect the skin, hair and mucuous membrane (from pollution, cold, chemical aggression, dehydration), restore stratum corneum barrier function, improve cutaneous visco-elastic properties and surface characteristics, enhance skin or hair sensory qualities (softness, smoothness) or look (radiance, shine), nourish dry skins and mucuous membranes or help sooth sensitive ones. We explored the advantages and limits of these methods.
\end{abstract}

Keywords: claims substantiation / cutaneous measurement / non invasive methods / clinical testing / cosmetics / lipids / shield effect

\begin{abstract}
Résumé - Méthodes non invasives permettant de mesurer in vivo l'efficacité de formules lipidiques en surface de la peau: avantages et limites. Les lipides sont des ingrédients essentiels des formules cosmétiques, et confèrent à ces produits des propriétés leur permettant d'améliorer l'aspect, les qualités sensorielles et les fonctions de la peau, des cheveux, des ongles et des muqueuses, soit en interagissant directement avec des composants de ces tissus, soit en promouvant la libération de l'ingrédient actif ou en formant une couche protectrice à sa surface. Mesurer l'efficacité de la composante lipidique des formules cosmétiques en surface de la peau est possible grâce à un large éventail de méthodes non invasives cliniques et instrumentales. Les mesures du sébum, de la température cutanée, de l'hydratation de surface, de la perte insensible en eau, de la topographie de surface de la peau, de la fermeté de la peau, de son élasticité, ou encore de sa couleur sont autant de moyens permettant d'évaluer la fonction protectrice notamment de lipides. Cette fonction protectrice, contre la pollution, le froid, les agressions environnementales, la déshydratation, par exemple, peut se mesurer par l'étude de la fonction barrière de la peau, ou de ses propriétés visco-élastiques, ou encore des caractéristiques sensorielles ou esthétiques de surface de la peau ou des cheveux (douceur, aspect lisse, éclat, brillance). Nous avons exploré les avantages et les limites de ces méthodes.
\end{abstract}

Mots clés : justification des allégations / mesures cutanées / méthode non invasives / études cliniques / cosmétiques / lipides / effet bouclier

\section{Introduction}

Lipids are major ingredients in cosmetics and play an essential role in cosmetics ability to improve skin, hair, nail and mucuous membranes aspect, sensory qualities and function. As lipids in cosmetics may be of different categories,

*Correspondence: elodieprestat-marquis@eurofins.com their behaviors to the skin vary. They can act either by interacting with skin components, enhancing active ingredient release or by spreading and forming a protective layer on its surface.

Measuring lipids efficacy to the skin is possible through a wide range of non-invasive clinical and instrumental methods. Measurements of sebum, temperature, hydration, transepidermal water loss, skin surface topography, skin firmness, elasticity, colour enable to assess the lipids ability to protect 


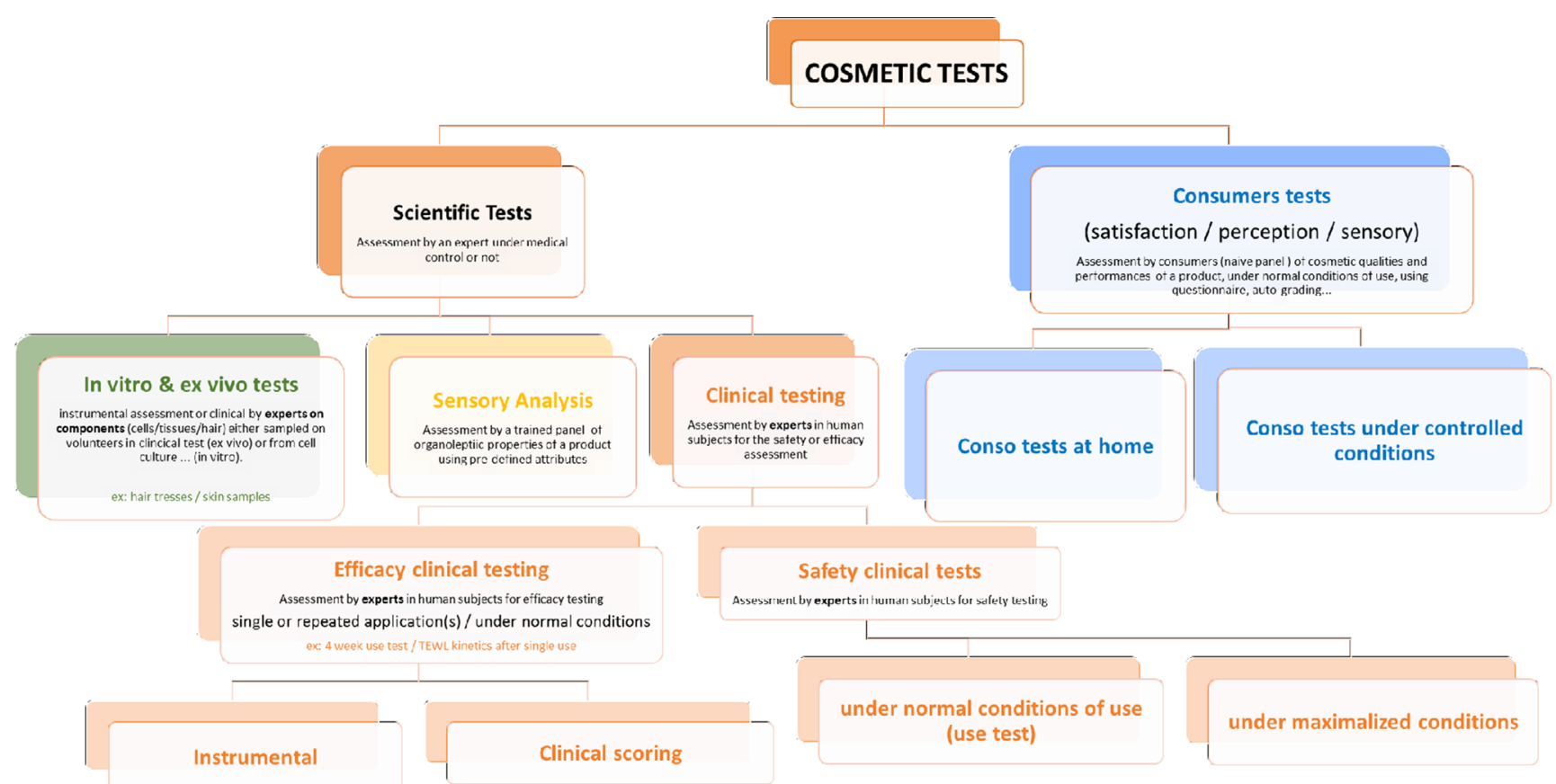

Fig. 1. Summary of the different categories of tests for Cosmetics efficacy and safety assessment.

the skin, hair and mucuous membrane (from pollution, cold, chemical aggression, dehydration), restore stratum corneum barrier function, improve cutaneous visco-elastic properties and surface characteristics, enhance skin or hair sensory qualities (softness, smoothness) or look (radiance, shine), nourish dry skins and mucuous membranes or help sooth sensitive ones.

We explored the advantages and limits of these methods. We present in this document the methods dedicated to the measure of the shield effect of fatty substances or formulae which contain them.

\section{Evaluation of cosmetics efficacy}

In compliance with the European Regulation (EC) No. 1223/2009, claims substantiation related to the cosmetics products efficacy must comply with six common criteria established in the European Commission text No. 655/2013 of $11 / 07 / 2013$. One of these criteria refers to evidences supporting claims (Règlement UE, 2013).

Evidential support:

- claims for cosmetic products, whether explicit or implicit, shall be supported by adequate and verifiable evidence regardless of the types of evidential support used to substantiate them, including where appropriate expert assessments;

- evidence for claim substantiation shall take into account state of the art practices;

- where studies are being used as evidence, they shall be relevant to the product and to the benefit claimed, shall follow well-designed, well-conducted methodologies (valid, reliable and reproducible) and shall respect ethical considerations;

- the level of evidence or substantiation shall be consistent with the type of claim being made, in particular for claims where lack of efficacy may cause a safety problem;
- statements of clear exaggeration which are not to be taken literally by the average end user (hyperbole) or statements of an abstract nature shall not require substantiation;

- a claim extrapolating (explicitly or implicitly) ingredient properties to the finished product shall be supported by adequate and verifiable evidence, such as by demonstrating the presence of the ingredient at an effective concentration;

- assessment of the acceptability of a claim shall be based on the weight of evidence of all studies, data and information available depending on the nature of the claim and the prevailing general knowledge the end users.

By "Evidential support", the legislator means all appropriate and verifiable evidence for claim effects. Concerning efficacy claims of cosmetics products, these evidential supports often interpretation of results from tests aiming to evaluate products efficacy. "Cosmetics tests" are all sort of investigations, in vivo, ex vivo and in vitro, aiming to measure, appreciate and evaluate the efficacy, the performance and characteristics of cosmetics products. Many approaches still exist, from the in vitro studies on cellular model to the consumers' perception tests by way of clinical testing on healthy adult subjects (Fig. 1).

Among cosmetics tests indicated by "clinical", only certain ones follow international standard methods (example: CEN, ISO...); such as tests aiming to evaluate the sun protection factor-SPF (ISO, 2010). Apart from these specific standards, the management of cosmetics clinical testing is guided by guidelines like the ones published by Cosmetics Europe and the evocation by regulations of "good practices" (Technical document on cosmetic claims, 2017). From these guidelines, the evaluation of cosmetics products efficacy must be carried out according to validated and reproducible methods and must follow a scientifically validated and adapted protocol which includes a sufficient number of volunteers (eligible on the base 
of relevant criteria of inclusion) and for which the results analysis must be based on reliable statistics methods. Implemented evaluation methods must consider the latest developments, must be inspired of published research or intern developments that followed reliable validation process in investigation centers, must be managed in controlled and standardized environments. Moreover, investigating teams who are in charge to lead the evaluation studies must be qualified, experimented, demonstrate professional integrity and must be trained to the international standards for ethics. In addition, the investigation center must have implemented a quality process which permits to ensure the reliability of processes and data from studies including a regular monitoring system.

Clinical evaluation protocols development is based on the capacity of Clinical Testing Laboratories Research \& Development teams to propose ways for cosmetic efficacy evaluation by integrating the knowledge of cutaneous investigation method, the understanding of biological action mechanisms of cosmetics products and marketing claims associated. The method must be adapted, sensitive enough to measure or detect a cosmetic effect and ideally, reflects the perception of consumers in use.

\section{Evaluation of cosmetics lipid components effects}

Cutaneous lipids are from sebaceous or keratinocyte origins. Lipids from sebaceous glands are forming the sebum, a complex combination of triglycerides, wax esters, squalene, fatty acids, cholesterol, cholesterol esters and diglycerides coating skin surface with: protective (physical and biological) and lubricant functions. Keratinocyte lipids form a matrix rich in fatty acids, cholesterol and ceramides playing a role on the cells of cutaneous surface involved in skin's barrier function.

The cosmetics lipid components are able to supplement or balance cutaneous lipids to play their different roles.

For example, different protocols have been developed in EUROFINS clinical tests laboratories in order to measure or appreciate the effects of these cosmetics formulas on the natural skin lipids. We will present here the experimental approaches implemented to appreciate the efficacy of the lipid formula to protect skin surface named the shield effect.

The shield effect of cosmetics formulae is in their capacity to form a lasting film on the skin surface in order to protect it against external phenomena (cold, pollution particles) or internal phenomena (transepidermal water loss). This efficacy can be appreciated via different non-invasive methods.

\subsection{Measurement of the product ability to form a durable protective layer on the skin surface}

Several products claim the capacity to form a protective layer on the skin surface but the measure of this efficacy is rarely realized in vivo. The physical presence of the product on the skin surface (protect layer) can be, for example, realized using a frictiometer. Indeed, the friction coefficient of skin surface changes in presence or absence of the product. So it's possible to identify, versus a non-treated area (control), if a product is long-lasting on the skin surface. The implemented methodology consists in the selection of a panel of healthy adult volunteers and an application of a standard quantity of product on their skin surface, on one or several delimited areas on the inner forearms. Measures are realized with a Frictiometer FR 700 (Courage \& Khazaka) on a non-treated area (bare skin) and on the treated area(s) before the application of the product and at different kinetic points (example: immediately and one hour after application). The presence of a product layer on the skin surface is highlighted by a variation of the friction coefficient. The product layer effective lasting in time is measured thanks to friction coefficient variation measurement several hours after application in comparison with the non-treated area (control). This methodology allowed to highlight the effective lasting of a balm at the laboratory. The limits of this method are mainly technical and are linked to the technician capacity to apply the product in homogenous layer allowing a replicable measure of the friction coefficient in different points of the application area. It is recommended to increase the number of areas and measures in order to be close to a representative average value. Each protocol must absolutely integrate a control area because the frictiometry measure is exploitable only by comparison with a non-treated area in this precise case.

\subsection{Measurement of the product ability to form a protective layer against the cold on the skin surface}

Lot of cosmetics claim a protective efficacy against extreme environmental conditions (cold, wind...). What about the evidence underlying these claims? Yet, the experimental measure of the cosmetic protective effect on the skin surface can be understood by the evaluation of the induced effect by cold contact. In this case, the aim of the method is to highlight that an exposure to cold produces a cutaneous parameters variation like the surface temperature or the skin color (mainly the red component). Then, the application of a cosmetic product before a cold exposure can attenuate significantly these effects. The developed method involves the recruitment of healthy adult subjects whose skin is exposed to a standardized cold source during a predetermined period. Then, measures are performed for the skin temperature (Thermometer Fluke 566) and for skin color (Spectro-photocolorimeter, Minolta CM600D). Immediately after the removal of standardized cold source applied for $5 \mathrm{~min}\left(4^{\circ} \mathrm{C}\right.$ to $7{ }^{\circ} \mathrm{C}$ according to the method), a decrease in the skin temperature is recorded as well as a skin blushing (increase in colorimetric parameter $\mathrm{a}^{*}$ ). This method allowed to show that preliminary application of a balm helps to limit the decrease in the skin surface temperature and the skin blushing in comparison with a control area which is no preliminary treated. The limits of this method are about ethics and technical. The exposure to cold can only be performed for short duration of time and the required cold can't be intense, provided that the test should not create feelings of discomfort (and even less burns). Besides, the measure must be realized in a quick way in order to respect the kinetics (measure performed "immediately after the cold removal"). 


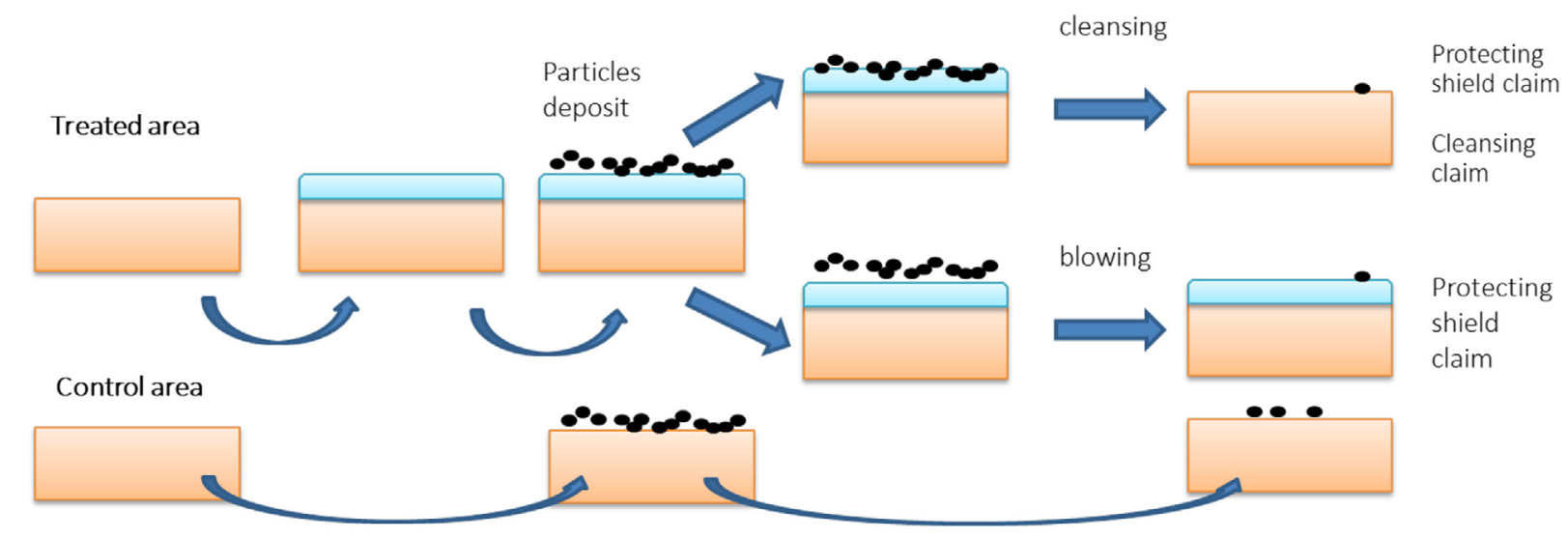

Fig. 2. Schematic representation of the particles deposit methodology.

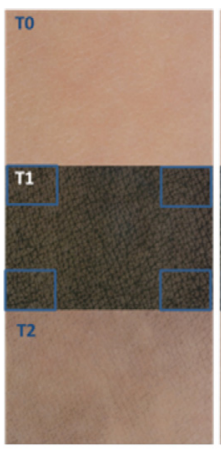

Normal skin

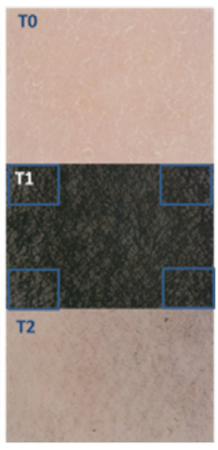

Dry skin

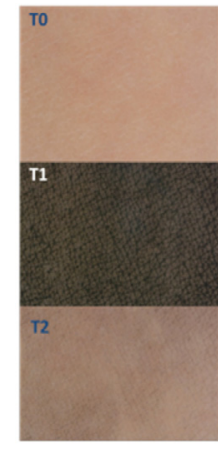

Young skin

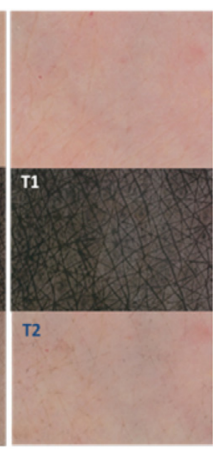

Older skin

Fig. 3. Particles distribution at the skin surface after deposit vary depending on squamous state at the skin surface (normal skin and dry skins compared) and the subject age (young and older skins compared).

\subsection{Measurement of the product ability to form a protective layer against the deposit of pollution particles on the skin surface}

Some cosmetics products claim to protect the skin against the injurious effects of the pollution. The action mechanisms are varied: one of them consists in creating a protective layer on the skin surface, like a shield limiting the bond even the infiltration of pollution particles through the skin. In order to apprehend the cosmetic product ability to form a protective film against the sediment of these pollution particles, another methodology has been developed in the Eurofins clinical laboratories. The aim of this method is to measure the adhesion of particles on the skin, with or without cosmetic product. The test is carried out on a panel of healthy adult volunteers. Two areas are delimited on the forearms of each volunteer: the first area is treated with the investigation product and the second area using as control (no product). A determined quantity of vegetal carbon particles with a similar granulometry like pollution particles (PM 2.5) is then applied on the two areas. These areas are then submitted to a standardized airstream or a standardized cleansing with a rinsed cleanser. At each step, a picture of the area is performed with the C-Cube camera

(Pixience), allowing then to measure the density of particles on each area and for each kinetic point, by picture analysis.

The comparison of the residual quantity of particles after cleansing or exposition at the airstream between the treated and the control areas enables to measure the product ability to limit the adhesion on the skin.

This method allowed to show the shield effect of care products (associated with the presence of fatty substances or polymers, for example) but also the cleansing efficacy on these particles by hygiene products (adaptation of the protocol without preliminary application of care product and by comparative cleansing of the area with a traditional cleansing and the investigate cleaning product). Many checkpoints for this methodology, mainly ethics and technical, have been comprehended over the development. They were on the choice of particles and the application methodology. Particles needed to be representative of pollution particles suspended in the air of polluted areas without presenting any toxicity. Another challenge was the choice of the adapted device to take pictures and analysis. The comparison of three equipments (VISA CR, C-Cube and Visioscan V98) as well as the different analytics approaches allowed to identify the final methodology which is the most suitable for test aims. The choice of the skin area on which to do the application was also tested, in order to compare the technique sensitivity on more or less dry skin, more or less young skin, the relief or various degrees of surface anisotropy (fine lines) that may have an effect on the powder distribution on the skin surface and thus on their analysis (Figs. 2 and 3).

\subsection{Measurement of the product capacity to form a protective layer against the transepidermal water loss}

When it comes to measure the protective efficacy of physical surface of a cosmetic product, the reference method consists in measurement of the product effect on a known biological parameter: the Trans-Epidermal Water Loss (TEWL). The TEWL is a passive diffusion phenomenon of the water through the skin from the outside of the organism. When the barrier function of the skin is altered, then the TEWL value increases. Strengthening the skin barrier function by the application of fatty substances leads to a decrease in the TEWL in comparison with a non-treated control area. Measurements can be done immediately after application of the product, 

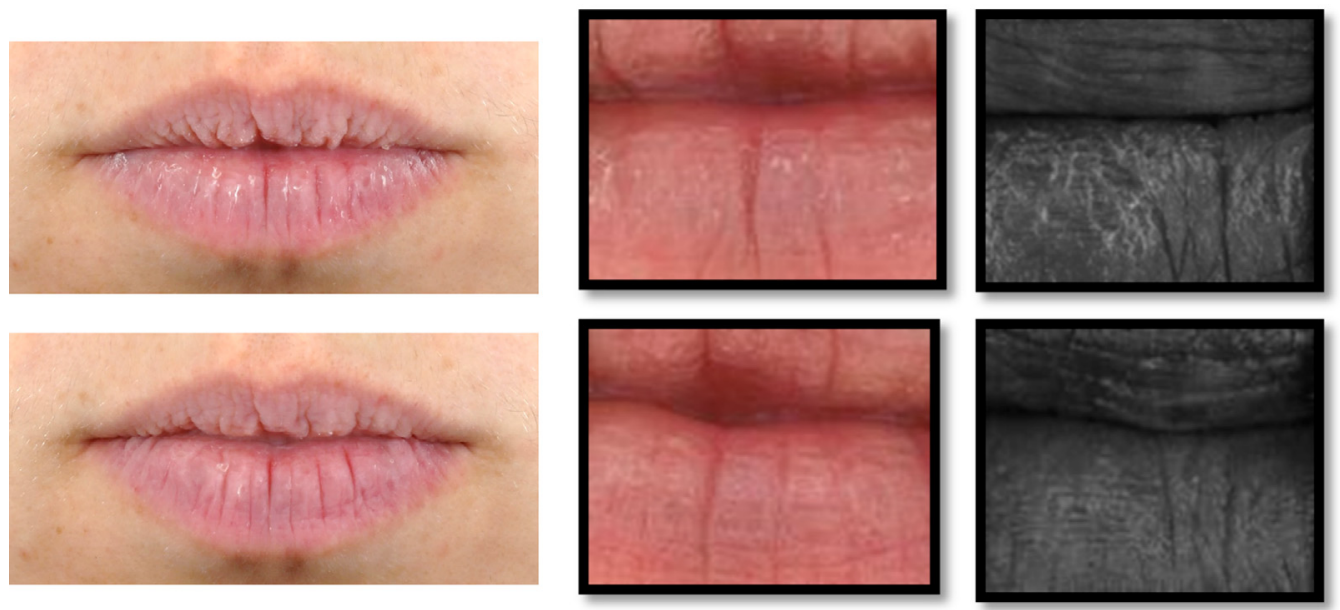

Fig. 4. Lips surface aspect improvement after repeated treatment with cosmetic balm for 2 weeks.

highlighting the physical shield effect of the product or on a bare skin after repeated applications of the product, highlighting then the "nourishing" even "restorative" function of the product, enhancing the skin barrier. The well-known limits of TEWL are mainly related to the large variability according to the temperature, the air movements and the hygrometry environment as well as the emotional state of the subject. It's therefore necessary to ensure a highly controlled environment for measuring and volunteer conditioning.

In addition, this effect can be illustrated by the macro shot picture of the area skin or mucous demonstrating the enhancement of the surface related to the treatment by fatty substances (Fig. 4).

\section{Conclusion}

Marketing claims constantly change, following cosmetic market trends and therefore constitute a permanent lever of innovation for formulation laboratories but also for expert teams conducting tests to support these claims in terms of regulations.

Protocols designed for the purpose of measuring the cosmetics products efficacy, and if appropriate, to feed cosmetics files with the necessary evidence to support the corresponding marketing claims, must therefore be developed dynamically, in response to market needs, but they also must imperatively be valid, reproducible and scientifically admissible.

We have presented here, for example, developments of non-invasive methods making it possible to understand the protective nature of the lipid formulas on skin surface in response to the claims related to the protective nature of the lipid cosmetics formulas (physical shield effect, protective effect against cold, pollution particle binding to the skin and Trans-Epidermal Water Loss).

\section{References}

Guidelines for the evaluation of the efficacy of cosmetics products May 2008.

ISO 24444:2010 (en) Cosmetics - Sun protection test methods - In vivo determination of the sun protection factor (SPF).

Règlement (UE) $n^{\circ} 655 / 2013$ de la commission du 10 juillet 2013 établissant les critères communs auxquels les allégations relatives aux produits cosmétiques doivent répondre pour pouvoir être utilisées.

Technical document on cosmetic claims; Agreed by the Sub-Working Group on Claims, (version of 3 July 2017).

Cite this article as: Prestat-Marquis E. 2018. Non-invasive in vivo methods to measure lipidic formulae efficacy at the skin surface: advantages and limits. OCL 25(5): D509. 